\title{
Top-Down Attentional Control in Parkinson's Disease: Salient Considerations
}

\author{
Roshan Cools ${ }^{1}$, Robert Rogers ${ }^{2}$, Roger A. Barker ${ }^{3}$, \\ and Trevor W. Robbins ${ }^{4}$
}

\begin{abstract}
Cognitive dysfunction in Parkinson's disease (PD) has been hypothesized to reflect a failure of cortical control. In keeping with this hypothesis, some of the cognitive deficits in PD resemble those seen in patients with lesions in the lateral pFC, which has been associated with top-down attentional control. However, there is no direct evidence for a failure of top-down control mechanisms in PD. Here we fill this gap by demonstrating disproportionate control by bottom-up attention to dimensional salience during attentional set shifting. Patients needed signifi-
\end{abstract}

\section{INTRODUCTION}

Parkinson's disease (PD) is accompanied by cognitive deficits, even in the earliest stages of the disease (Cools, 2006; Dubois et al., 1995; Owen et al., 1992, 1995; Brown \& Marsden, 1988b). Some of these deficits resemble those seen in patients with lesions in the lateral pFC (Owen et al., 1992, 1995). For example, both PD and lateral frontal lesion patients exhibit a set-shifting deficit on Wisconsin Card Sort-like tests (Owen, Roberts, Polkey, Sahakian, \& Robbins, 1991; Taylor, Saint-Cyr, \& Lang, 1986; Cools, Van Den Bercken, Horstink, Van Spaendonck, \& Berger, 1984; Lees \& Smith, 1983; Bowen, Kamienny, Burns, \& Yahr, 1975; Milner, 1963, 1964), such as the intradimensional/ extradimensional (ID/ED) set-shifting paradigm (Cools, Barker, Sahakian, \& Robbins, 2001b; Owen et al., 1993; Downes et al., 1989). Set-shifting impairment and other frontal lobe-like abnormalities have been associated with a failure of top-down control mechanisms (Miller \& D'Esposito, 2005; Miller \& Cohen, 2001; Dias, Robbins, \& Roberts, 1996; Milner, 1963, 1964). Although top-down control mechanisms are ill defined, they generally refer to selective attention processes that enable goal-directed, internally driven decisions and help the organism to overcome externally driven decisions based on stimulus salience and novelty (Gazzaley \& D'Esposito, 2007). The

\footnotetext{
${ }^{1}$ Donders Institute for Brain, Cognition, and Behavior, Radboud University Nijmegen Medical Centre, The Netherlands, ${ }^{2}$ University of Oxford, UK, ${ }^{3}$ Cambridge Centre for Brain Repair, UK, ${ }^{4}$ University of Cambridge, UK
}

cantly more trials to criterion than did controls when shifting to a low-salient dimension while, remarkably, needing significantly fewer trials to criterion than did controls when shifting to a highsalient dimension. Thus, attention was captured by bottom-up attention to salient information to a greater extent in patients than in controls. The results provide a striking reinterpretation of prior set-shifting data and provide the first direct evidence for a failure of top-down attentional control, resembling that seen after catecholamine depletion in the $\mathrm{pFC}$.

hypothesis that PD is characterized by a failure of top-down control is consistent with classic theorizing about setshifting deficits in PD, according to which they reflect problems with "internal" attentional control, leading to excessive guidance of behavior by "external cues" (Brown \& Marsden, 1988a; Cools et al., 1984; Barbeau, 1973). However, there is no direct evidence for a failure of top-down control underlying set-shifting deficits in PD. Here we fill this gap by demonstrating disproportionate control by bottom-up attention to dimensional salience during attentional set shifting.

In the attentional set-shifting paradigm, subjects discriminate between two patterns according to one of two stimulus dimensions (e.g., shapes or lines). During the critical extradimensional set-shifting (EDS) stage of the task, subjects shift their attention from a previously relevant dimension to a newly relevant dimension. Performance generally declines at these EDS stages relative to the earlier set formation and maintenance stages and disproportionately so in PD patients. Here we manipulated the bottom-up salience of the stimulus dimensions. Dimensional salience was established using a sorting task in a separate experiment with young healthy volunteers. This separate study (reported below) revealed that the salience (or attentiondrawing capacity) of the shape and color of a stimulus was greater than that of the angle of a stimulus. Accordingly, in the subsequent set-shifting experiment, patients and agematched controls were assigned to either a high-salient shape/color condition or a low-salient angle/color condition (relevant dimension italicized). This design allowed us to test whether the set-shifting deficit in PD depends 
on bottom-up dimensional salience and, thus, reflects a failure of control by top-down attention.

In addition, the design also enabled us to investigate an alternative hypothesis regarding the mechanism underlying the set-shifting deficit in PD. This alternative hypothesis is based on previous data (Slabosz et al., 2006; Owen et al., 1993; but see Myers et al., 2003; Gauntlett-Gilbert, Roberts, \& Brown, 1999; Van Spaendonck, Berger, Horstink, Borm, \& Cools, 1995), which have suggested that the set-shifting deficit in PD reflects enhanced learned irrelevance (LI). Learned irrelevance refers to an inability to attend to and learn about information that was previously irrelevant (Mackintosh, 1975) and has been contrasted with perseveration (PE), that is, an inability to disengage from a previously relevant dimension. Owen et al. (1993) argued that set-shifting deficits must result either from enhanced LI or from PE. To investigate this issue in PD, they decomposed the original attentional set-shifting paradigm into two tasks. At the postshift stage of the LI task, the previously relevant dimension was replaced with a novel dimension. Accordingly, PE was not possible. Any set-shifting deficit, they argued, must reflect enhanced LI. Conversely, at the postshift stage of the PE task, the previously irrelevant dimension was replaced. Any set-shifting deficit in this task could not reflect enhanced LI but must rather reflect PE. Mild medicated PD patients performed poorly only on the LI task while performing like normal controls on the PE task. This initial study, which suggested that the set-shifting deficit in (medicated) PD reflects enhanced LI, was followed up by a series of similar studies. Some of these replicated the originally obtained selectively enhanced LI (Slabosz et al., 2006; Lewis, Slabosz, Robbins, Barker, \& Owen, 2005). However, other studies observed findings that were not consistent with enhanced LI (Myers et al., 2003; Gauntlett-Gilbert et al., 1999; Van Spaendonck et al., 1995). Therefore, we reasoned that the deficit might surface only in certain conditions. Specifically, we hypothesized that enhanced LI might be particularly pronounced, when demands for suppression of the irrelevant dimension are high. Thus, LI would be a function of the degree to which such irrelevant information was processed before shifting, that is, of the salience of preshift (ir)relevant dimensions.

In sum, the aim of the present study was to assess whether the set-shifting deficit in PD reflects (i) abnormal control by bottom-up salience or (ii) enhanced LI. To this end, we employed a $2 \times 2$ factorial design with the following four conditions: a high-salient LI task, a low-salient LI task, a high-salient PE task, and a low-salient PE task (Table 1). If the set-shifting deficit reflects abnormal control by bottom-up salience, then it would surface as a salience-dependent deficit in the PE condition, in which salience was manipulated at the postshift stages (Table 1). By contrast, if the set-shifting deficit reflects enhanced LI, then it would surface as a salience-dependent deficit in the LI condition, in which salience was manipulated at the preshift stages.
Table 1. Conditions

\begin{tabular}{lccl} 
Condition & Task & Preshift Dimensions & Postshift Dimensions \\
\hline Low salient & PE & Color, number & Angle, color \\
High salient & PE & Color, number & Shape, color \\
Low salient & LI & Angle, color & Color, number \\
High salient & LI & Shape, color & Color, number \\
\hline
\end{tabular}

Relevant dimensions are italicized.

The results provide clear support for the former and not the latter hypothesis. The deficit was modulated by salience in the PE condition, and not in the LI condition. These data suggest that the deficit in tasks of LI observed in previous studies might actually reflect disproportionate control by bottom-up attention to a salient dimension. Indeed the previously used tasks confounded LI with dimensional salience, given that they required subjects to attend to a familiar, thus less salient, dimension and to ignore a novel, thus more salient, dimension. Accordingly, the LI deficit might have originated from an inability to overcome capture by a highly salient, currently irrelevant dimension due to a failure to exert top-down control. Such an account differs qualitatively from the alternative hypothesis that it reflects an inability to overcome enhanced LI specifically associated with the previously irrelevant, now relevant dimension.

\section{METHODS}

\section{Subjects}

Eighty-two mild to moderate PD patients (31 women) (as defined using UK Parkinson's Disease Brain Bank criteria) and 50 age- and IQ-matched elderly control subjects (30 women) participated in the experiment. The Hoehn and Yahr stage ranged from I to IV in the on medication state (where applicable) (mean \pm SEM $=2.0 \pm 0.06 ; 12$ stage 1.0, 9 stage $1.5,39$ stage $2.0,13$ stage 2.5 , 8 stage 3 , and 1 stage 4). All, except seven never-medicated patients (two in the high-salient PE group, two in the low-salient LI group, and three in the high-salient LI group), were receiving daily L-dopa preparations, dopamine receptor agonists, and/or selegiline (a monoamine oxidase inhibitor). Six patients received anticholinergic medication (one in the high-salient PE group, three in the low-salient LI group, and two in the high-salient LI group). Three patients received antidepressant medication (all in the highsalient PE group). All were stable on their doses for at least 3 months before testing. None of the patients were demented [Mini-Mental State Examination (MMSE) >24] or clinically depressed.

Subjects were randomly assigned to one of the four conditions (see below and Table 1). Table 2 shows the number of subjects attempting the critical EDS stage of each condition and the number of subjects starting each condition. 
Table 2. Sample Sizes

\begin{tabular}{lccc}
\hline Condition & Task & Patients & Controls \\
\hline Low salient & PE & $15 / 15$ & $13 / 13$ \\
High salient & PE & $15 / 15$ & $13 / 14$ \\
Low salient & LI & $23 / 27$ & $11 / 12$ \\
High salient & LI & $25 / 25$ & $11 / 11$ \\
\hline
\end{tabular}

Data represent the total number of subjects attempting the critical EDS stage/the total number of subjects starting the task.

Demographic characteristics and performance on background tests of pattern and spatial recognition memory (from the CANTAB battery [Sahakian et al., 1988]); the Beck Depression Inventory (BDI [Beck, Ward, Mendelson, Mock, \& Erbaugh, 1961]); the MMSE (Folstein, Folstein, \& McHugh, 1975); and the National Adult Reading Test (NART [Nelson, 1982]) are presented in Table 3. There were no significant differences between patients and controls, except on the BDI, $F(1,113)=20.5, p<.001$, where patients had higher scores than controls. Patients also made more errors on the spatial recognition memory test than did controls, $F(1,116)=6.6, p=.01$. However, there was no three-way interaction between group, task, and dimensional salience on any of these measures, indicating that the critical interaction effects of interest (see below) do not reflect differences between subgroups (divided across the different task conditions) in terms of performance on the background tests. Similarly there were no two-way interactions between task and dimensional salience, that is, between the four PD patient subgroups [Task $\times$ Salience interactions for age, $F(1,78)=0.02$; NART, $F(1,78)=0.2$; UPDRS, $F(1,78)=0.06$; Hoehn and Yahr, $F(1,78)=0.3$ ]. Together with the relatively normal performance of the patients on the initial stages of the attentional set-shifting tasks (see below and Figure 4) [for example, no main effect of group, $F(1,124)=0.4, p=.5$, and no Group $\times$ Stage $\times$ Salience interaction, $F(1,124)=0.06, p=.8$, for trials to criterion (TTC) at the simple discrimination (SD) stage], these data show that performance at the critical EDS stages of the tasks could be examined in the context of relatively matched global cognitive function.

\section{Procedure}

A $2 \times 2$ factorial between-subjects design was employed with four separate conditions (see Table 1). Like the standard CANTAB ID/ED set-shifting paradigm, this paradigm consisted of a series of stages (see Figures 1 and 2), starting with an SD task. During this SD stage, subjects were presented two abstract one-dimensional visual patterns (i.e., two shapes) and had to learn by trial and error, based on performance feedback, which of the two patterns was

Table 3. Demographic and Background Characteristics

\begin{tabular}{|c|c|c|c|c|c|c|c|}
\hline Age & Hoebn and Yabr & UPDRS & $N A R T$ & MMSE & $B D I$ & $\begin{array}{c}\text { Pattern } \\
\text { Recognition Memory }\end{array}$ & $\begin{array}{c}\text { Spatial } \\
\text { Recognition Memory }\end{array}$ \\
\hline \multicolumn{8}{|l|}{ Perseveration } \\
\hline \multicolumn{8}{|l|}{ Low salient } \\
\hline PD $62.1(1.8)$ & $1.77(0.14)$ & $29.4(3.8)$ & $110.3(2.8)$ & $29.3(0.3)$ & $9.3(1.6)$ & $21.1(0.8)$ & $15.5(0.5)$ \\
\hline CS 61.8 (2.6) & $\mathrm{Na}$ & $\mathrm{Na}$ & $116.9(1.7)$ & $29.2(0.3)$ & $5.1(0.7)$ & $21.1(0.8)$ & $16.3(0.8)$ \\
\hline \multicolumn{8}{|l|}{ High salient } \\
\hline PD 64.5 (1.9) & $2.07(0.15)$ & $37.0(4.6)$ & $111.7(2.4)$ & $28.7(0.4)$ & $10(1.6)$ & $19.7(0.8)$ & $14.3(0.5)$ \\
\hline CS $62.3(2.2)$ & $\mathrm{Na}$ & & $113.9(2.4)$ & $28.4(0.6)$ & $3.3(1.0)$ & $21.2(0.6)$ & $16.2(0.6)$ \\
\hline \multicolumn{8}{|c|}{ Learned irrelevance } \\
\hline \multicolumn{8}{|l|}{ Low salient } \\
\hline PD $61.8(2.2)$ & $1.98(0.12)$ & $34.8(3.1)$ & $115.6(1.6)$ & $28.9(0.24)$ & $9.1(1.1)$ & $20(0.5)$ & $15.4(0.4)$ \\
\hline CS $61.4(3.0)$ & $\mathrm{Na}$ & & $115(2.0)$ & $28.5(0.4)$ & $5.9(1.4)$ & $20.6(1.1)$ & $14.8(1.1)$ \\
\hline \multicolumn{8}{|l|}{ High salient } \\
\hline PD $63.6(1.5)$ & $2.12(0.12)$ & $44.3(4.0)$ & $115.1(1.88)$ & $29.4(0.3)$ & $7.8(1.1)$ & $19.7(0.7)$ & $14.3(0.4)$ \\
\hline CS 59.7 (2.7) & $\mathrm{Na}$ & & $118.5(1.6)$ & $29.3(0.2)$ & $3(0.7)$ & $19.2(1.2)$ & $16.8(0.6)$ \\
\hline
\end{tabular}

Data represent means (SEM); eight control data points missing for the spatial recognition memory test; four control data points missing for the pattern recognition memory test; four patient data points and seven control data points missing for the BDI (Beck et al., 1961); two patient data points and seven control data points missing for the MMSE (Folstein et al., 1975); and one control data point missing for the National Adult Reading Test (Nelson, 1982). PD = Parkinson's disease; CS = control subjects. 


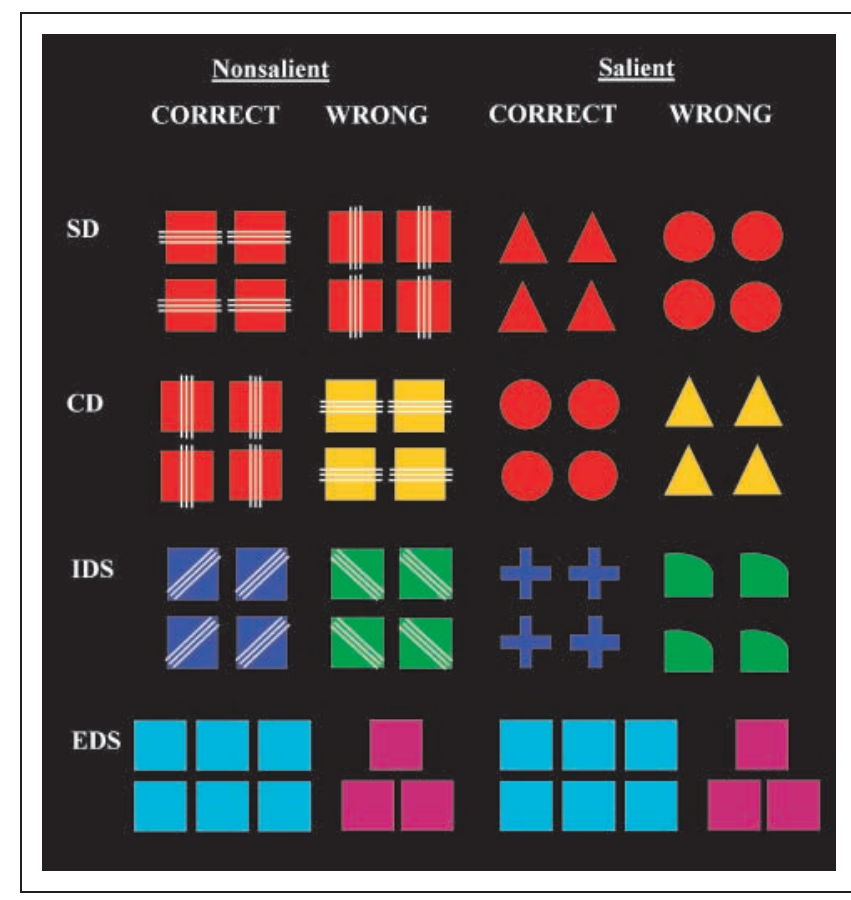

Figure 1. Correct and incorrect stimulus exemplars in the high-salient (right panel) and the low-salient (left panel) conditions of the LI tasks.

correct. After subjects obtained a learning criterion of six consecutive correct responses, the task proceeded to the next stage, the SD reversal stage. In this stage, the stimulusreward contingencies were reversed so that the previously correct pattern now became incorrect, whereas the previously incorrect pattern became correct. Subjects had to shift responding from one exemplar pattern to the other exemplar pattern. In the third stage, the compound discrimination (CD) stage, a second dimension (e.g., color), was introduced, but subjects had to ignore this new dimension and continue attending to the previously relevant dimension. Following a fourth CD reversal (CDR) stage, in which the stimulus-reward contingencies of the original dimension reversed once more, the task proceeded to an intradimensional shift (IDS) stage, at which new exemplars of both dimensions were introduced. Subjects still had to respond according to the original dimension (e.g., shape) and to ignore the other irrelevant dimension (e.g., color). The sixth stage, the intradimensional reversal (IDR) stage, in which the stimulus-reward contingencies of the original dimension were reversed, was followed by the critical EDS stage, at which point subjects had to shift attentional set and start responding according to a newly relevant dimension (e.g., number). A final reversal of stimulus-reward contingencies occurred at the eighth ED reversal stage.

For each stage, the learning criterion was set to six consecutive correct trials, after which the task proceeded to the next stage. If this criterion was not reached after 50 trials, then the test terminated automatically. A "total change" design was employed in which both the IDS and the EDS were made in the presence of novel exemplars of the two dimensions. The task took approximately $25 \mathrm{~min}$.

Subjects were assigned to either an LI task or a PE task. As in the study by Owen et al. (1993), the LI task (Figure 1) emphasized the requirement to shift attention to the previously irrelevant dimension, whereas the PE task (Figure 2) emphasized the requirement to shift attention away from the previously relevant dimension. In the LI task, the preshift relevant dimension was replaced with a novel dimension at the EDS stage to prevent PE. The preshift irrelevant dimension became the relevant dimension at the EDS stage of the LI task. In the PE task, the preshift irrelevant dimension was replaced with a novel dimension at the EDS stage to prevent LI. The preshift relevant dimension became the irrelevant dimension at the EDS stage of the PE task.

Subjects were assigned to either the high-salient shape/ color condition (Figures 1 and 2, right panels) or the lowsalient angle/color condition (Figures 1 and 2, left panels). Dimensional salience was manipulated at preshift stages in the LI tasks and at postshift stages in the PE tasks, so that in the LI tasks, any difference in performance between conditions at the EDS stage can only reflect differences in the pre-EDS stages.

We assessed dimensional salience in a separate experiment by using a speeded classification task, which required subjects to sort one- and two-dimensional sample stimuli to one of two test stimuli according to a one-dimensional rule as fast as possible (Gottwald \& Garner, 1975; Garner \& Felfoldy, 1970). For the two-dimensional stimuli, the

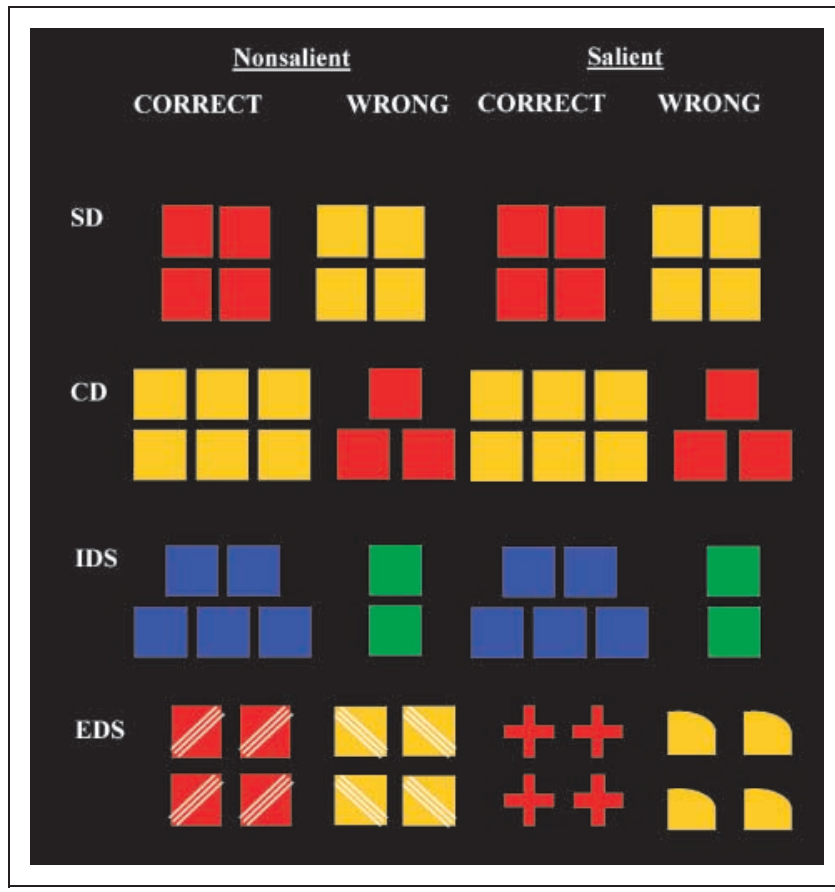

Figure 2. Correct and incorrect stimulus exemplars in the high-salient (right panel) and the low-salient (left panel) conditions of the PE tasks. 


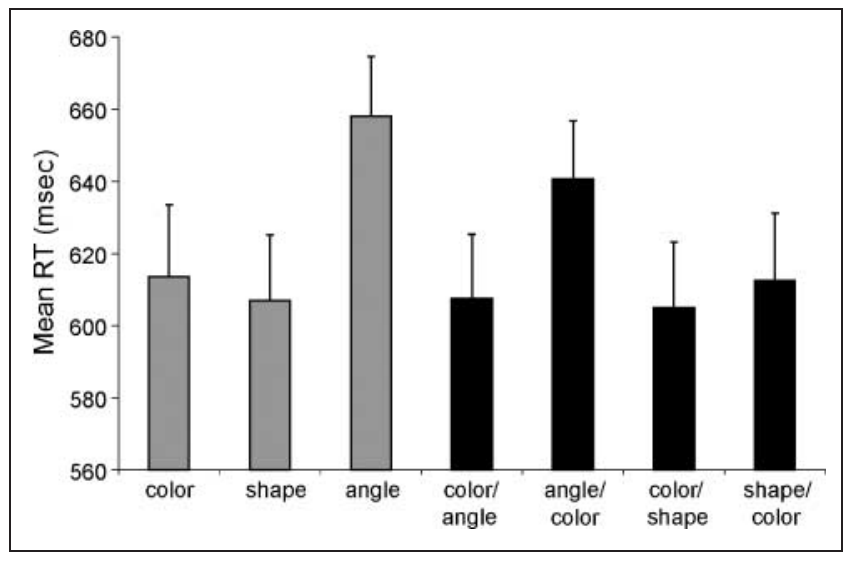

Figure 3. Means of median RTs (msec) are shown for each of the seven sorting tasks. Error bars represent standard errors of the mean.

dimensions were paired orthogonally, thus requiring selective attention to the currently relevant dimension. Comparison of the sorting of the different two-dimensional stimuli with each other as well as with the sorting of the one-dimensional stimuli enabled us to test the hypothesis that the dimension angle was less salient than the dimensions color and shape. Specifically, we predicted that the sorting of one-dimensional angle stimuli would be more difficult than the sorting of one-dimensional color and shape stimuli. In addition, we predicted that the sorting of two-dimensional angle/color stimuli (relevant dimension italicized) would be more difficult than the sorting of twodimensional color/angle stimuli. Conversely, we predicted that there would be no difference between sorting shape/ color and color/shape stimuli. Twenty-three nonmedicated young healthy volunteers (Cambridge undergraduates) participated in this experiment. Each subject performed seven blocks, each block consisting of twenty trials. The first three blocks required the sorting of one-dimensional stimuli according to color, shape, or angle (order randomized between subjects). The last four blocks required the sorting of two-dimensional stimuli according to (i) color while ignoring angle, (ii) angle while ignoring color, (iii) shape while ignoring color, and (iv) color while ignoring shape. Subjects were presented a stimulus inside a box located at
Figure 4. The number of TTC for each of the eight stages of the PE tasks (A) and the LI tasks (B) as a function of dimensional salience $(\mathrm{a} / \mathrm{c}=$ angle/color [low salient]; s/c = shape/color [high salient]) and group $(\mathrm{PD}=$ patients with Parkinson's disease; CS = age- and education-matched controls). SD = simple discrimination; SDR = simple discrimination reversal;

$\mathrm{CD}=$ compound discrimination; $\mathrm{CDR}=$ compound discrimination reversal; IDS = intradimensional shift;

IDR $=$ intradimensional reversal; EDS $=$ extradimensional set shift; EDR = extradimensional reversal. Error bars represent standard errors of the mean.
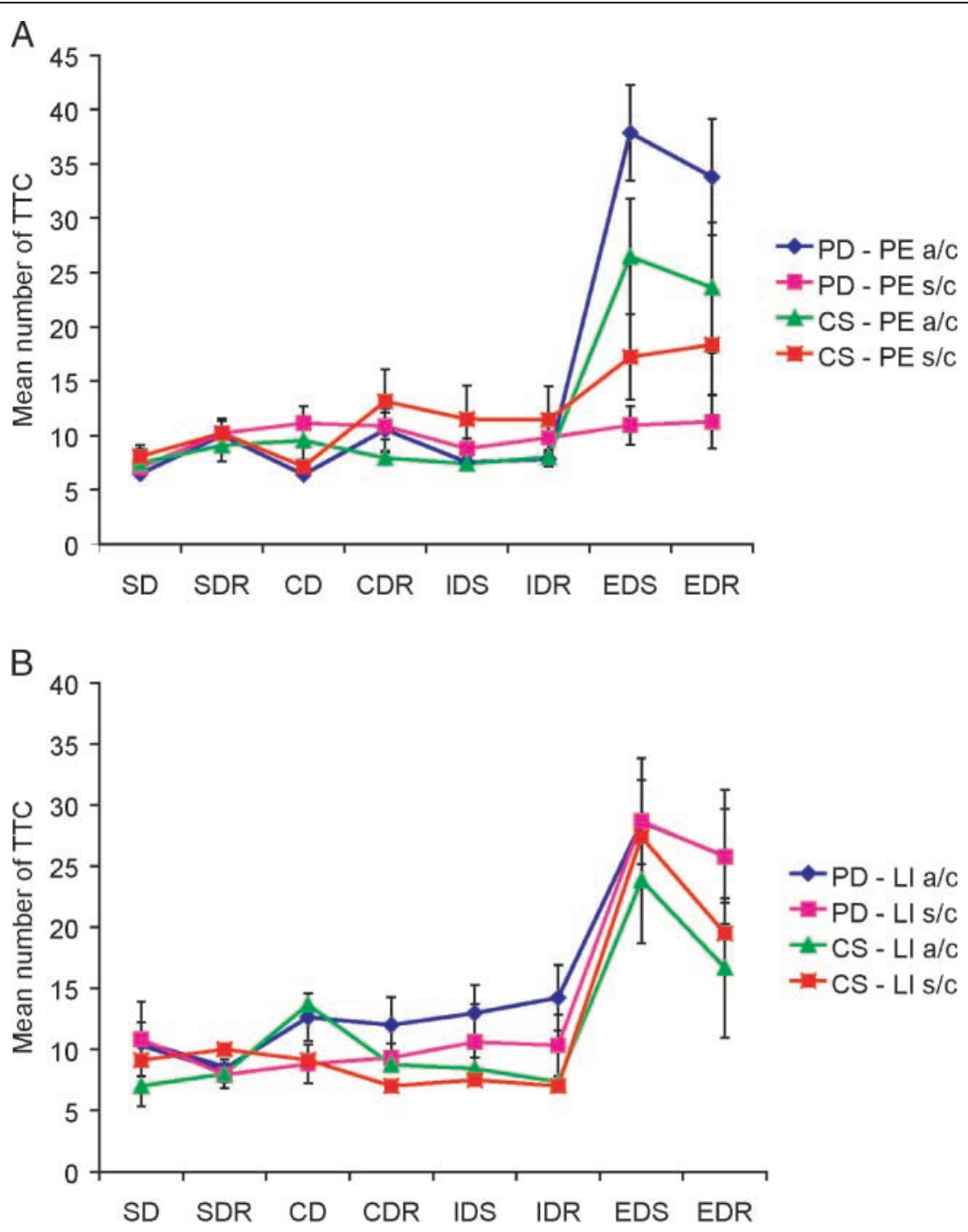
the top of the computer screen and were asked to sort it into one of two lower boxes by touching that box as fast as possible according to an instructed task dimension (e.g., shape).

\section{Stimuli and Material}

An Advantech PPC-120T-RT computer with a touch-sensitive screen for recording responses was used as a testing machine. On each trial of the adapted ID/ED set-shifting paradigm, two stimuli were presented randomly within two of four boxes ( $10.5 \mathrm{~cm}$ wide and $5 \mathrm{~cm}$ high) on a black background. The distance between the top and the bottom box was $7 \mathrm{~cm}$. The distance between the left and the right box was $3.8 \mathrm{~cm}$. Angle stimulus pairs were always vertical and horizontal or $45^{\circ}$ clockwise and $45^{\circ}$ anticlockwise (Figure 1). Shape stimulus pairs were always a circle and a triangle or a cross and a rough quarter of a circle. The default shape value (used for angle/color and color/number stimuli) was a square. The numbers of exemplars presented for number stimuli were always three and six or two and five (Figure 1). The default number value was four. Color stimulus pairs were always red and orange, blue and green, or cyan and magenta. The default value was red. The two exemplars of the irrelevant dimension were always paired pseudorandomly with those of the relevant dimension so that the same pairing did not occur on more than three successive trials. The exact sequences of stimuli are given in Figures 1 (LI) and 2 (PE). An incorrect response was followed by the word "wrong" in red, presented in the center of the screen. The word was accompanied by the outline of the touched box turning red and a low-pitch tone. A correct response was followed by the word "correct" in green; the touched box turning green and a high-pitch tone.

\section{Data Analysis}

Sorting Task

Median RTs were calculated and preferred over mean RTs due to reduced sensitivity to outliers. They were submitted to a series of repeated measures ANOVAs, after $\log 10$ transformation (as is appropriate when data are positively skewed as is the case with RT data; Howell, 1997 , p. 325) and exclusion of RTs >2000 msec, error trials, and the first trial of each block.

\section{Attentional Set-shifting Task}

On the basis of our a priori hypothesis that attentional set shifting in PD depends on demands for selective attention (i.e., dimensional salience), we focused our analyses on the two stages of the paradigm that were expressly designed to investigate these processes: the CD stage, where the second irrelevant dimension is first introduced, and the EDS stage, where subjects have to shift between dimensions. The main performance measure was the mean number of trials to criterion (TTC), which were square root transformed $[\sqrt{ } x+0.5]$ to stabilize variances as is appropriate when the mean is proportional to the variance, for example, when data are in the form of counts (Howell, 1997, p. 327). These data were analyzed using repeated measures ANOVAs, with stage as the within-subject factor and group (patients vs. controls), dimensional salience (angle/color vs. shape/color), and task (PE vs. LI) as between-subject factors. Partial eta squared values $\left(\eta^{2}\right.$; calculated using SPSS) represent the proportion of the total variance that is attributed to the effect and were calculated as the ratio of the effect variance $\left(\mathrm{SS}_{\text {effect }}\right)$ to the total variance $\left(\mathrm{SS}_{\text {total }}\right)$. For simplicity, the error data and the failure rate data are not reported, as they revealed the same pattern of results.

\section{RESULTS}

\section{Effect of Dimensional Salience on Speeded Sorting}

The performance of young volunteers $(n=23)$ on the seven sorting tasks is presented in Figure 3. Subjects sorted one-dimensional angle-stimuli significantly more slowly relative to both one-dimensional color stimuli, $F(1,22)=13.4, p=.001, \eta^{2}=0.4$, and one-dimensional shape stimuli, $F(1,22)=19.0, p<.0001, \eta^{2}=0.5$, whereas there was no difference in sorting speed between the one-dimensional shape and the color blocks, $F(1,22)=0.2, p=.6, \eta^{2}=0.01$. In addition, subjects sorted angle/color stimuli significantly more slowly than color/angle stimuli, $F(1,22)=12.5, p=.005, \eta^{2}=0.4$. Conversely, there was no difference in sorting speed between the shape/color and color/shape blocks, $F(1,22)=$ $0.4, p=.5, \eta^{2}=0.02$. These results confirmed our predictions and indicated that the dimension angle was less salient than the dimensions color and shape.

\section{Effects of Dimensional Salience on Attentional Set-shifting in PD}

In Figure 4, we present shifting difficulty as a function of group, dimensional salience, and task (LI vs. PE). As expected, the performance pattern of patients was significantly different from that of controls, particularly at the

Table 4. Mean TTC at the Compound Discrimination Stage

\begin{tabular}{lcr}
\hline & PD Patients & Controls \\
\hline Low-salient PE & $6.4(0.2)^{\mathrm{a}}$ & $9.5(1.9)$ \\
High-salient PE & $11.1(1.5)$ & $7.1(0.6)$ \\
Low-salient LI & $12.9(2.0)^{\mathrm{b}}$ & $14.7(3.6)$ \\
High-salient LI & $8.2(0.6)$ & $9.5(0.8)$ \\
\hline Values represent means (SEM). & \\
${ }^{\mathrm{a}}$ Significantly different from the high-salient PE condition. & \\
${ }^{\mathrm{b}}$ Significantly different from the high-salient LI condition.
\end{tabular}


EDS stage, but the difference depended on dimensional salience and task, as evidenced by a significant Group $\times$ Stage $\times$ Salience interaction, $F(1,124)=5.9, p=.02, \eta^{2}=$ 0.05 , and a strong trend toward a significant four-way Group $\times$ Stage $\times$ Salience $\times$ Task interaction, $F(1,124)=$ $3.7, p=.056, \eta^{2}=0.03$. There was also a main effect of stage, $F(1,124)=100.1, p<.0001, \eta^{2}=0.45$, and a Group $\times$ Stage $\times$ Task interaction, $F(1,124)=19.6, p<$ $.0001, \eta^{2}=0.14$. Breakdown of the four-way interaction revealed that the Group $\times$ Stage $\times$ Salience interaction was significant for the PE task, $F(1,53)=9.3, p=.004, \eta^{2}=0.15$, but not for the LI task, $F(1,71)=0.1, p=.7, \eta^{2}=0.002$. In fact, there was no effect of group on the LI task at all at any stage (all $p s>.2)$.

Further breakdown of the significant Group $\times$ Stage $\times$ Salience interaction in the PE task revealed that patients needed significantly more TTC at the EDS stage (relative to the CD stage) in the angle/color condition than in the shape/color condition (a Stage $\times$ Salience interaction), $F(1,28)=40.6, p<.0001, \eta^{2}=0.6$ (Figure 4). This effect of dimensional salience was not present in controls, $F(1,25)=0.5, p=.5, \eta^{2}=0.02$. A group difference was also evident when patients and controls were compared directly for the conditions separately: Patients needed more TTC at the EDS stage (relative to the CD stage) than controls in the angle/color condition (a significant Group $\times$ Stage interaction), $F(1,26)=4.1, p=.05, \eta^{2}=$ 0.14 . Conversely, they needed fewer TTC than controls in the shape/color condition, $F(1,27)=5.8, p=.023, \eta^{2}=$ 0.18 (Figure 4).

Simple main effects analyses revealed a significant effect of salience in the patient group at both the EDS and the CD stages (Figure 4A and Table 4): At the EDS stage, patients in the angle/color PE condition needed significantly more TTC than patients in the shape/color PE condition, $F(1,28)=32.0, p<.0001, \eta^{2}=0.5$. Conversely, at the CD stage, patients in the angle/color PE condition needed significantly fewer TTC than patients in the shape/color condition, $F(1,28)=11.7, p=.002, \eta^{2}=0.3$. These simple main effects of salience were not significant in the control group (both $p>.2$ ).

The significant effect of salience at the CD stage of the PE task in the patient group (Figure 4A and Table 4) was surprising because the CD stages of the two PE conditions were identical (the locus of the salience manipulation was at postshift stages). Accordingly, this difference likely reflects a sample bias. However, there were no significant differences between the shape/color and the angle/color patient groups on any of the background neuropsychological tests (Table 3 ). Nevertheless, we considered the possibility that the effect of dimensional salience at the EDS stage in the patients reflected an incidental difference in set formation and/or maintenance at the CD stage. Indeed, one might expect that it is easier to shift away from a poorly formed and maintained set than to shift from a well-formed and well-maintained set. We rejected this hypothesis based on the following two findings. First, performance was well matched between the two patient groups at the IDS and the IDR stages (both $F \mathrm{~s}<1.3$, both $p s>3$ ), which directly preceded the EDS stage. This finding indicates that there was no incidental difference in set-maintenance immediately before shifting. Second, the critical significant difference in EDS performance between patients in the angle/color condition and patients in the shape/color condition remained highly significant even after covarying for performance at the CD stage, $F(1,27)=19.9, p<.0001, \eta^{2}=0.4$.

In summary, these data show that the parkinsonian EDS deficit depends on dimensional salience. Patients exhibited a significant deficit when shifting to a low-salient dimension. By contrast, they exhibited a significant benefit when shifting to a high-salient dimension. These salience-dependent effects were restricted to the postshift stages of the PE task, in which the salience of the previously relevant dimension remained constant. Thus, the salience-dependent effects cannot reflect disproportionate PE to that previously relevant dimension but must reflect abnormal differential processing of the novel, newly relevant dimensions angle versus shape. The saliencedependent effects of PD did not extend to postshift stages of the LI task, indicating that they also do not reflect enhanced LI.

\section{DISCUSSION}

We demonstrate that the attentional set-shifting deficit in mild medicated PD patients was present only when they were required to shift to a low-salience attentional set. By contrast, patients were significantly better than controls at shifting to a high-salience attentional set. This performance pattern shows that impaired set shifting in PD does not reflect nonspecific changes in motivation, arousal, or fatigue. Instead the results reveal that, after shifting, attention was captured by salient information to a greater extent in patients than in controls, indicative of exaggerated bottom-up and/or attenuated top-down attentional control in PD. We hypothesize that this disproportionate bottom-up control is a result of a failure of top-down control. This hypothesis builds on the principle of competitive interactions between top-down and bottom-up control processes (Einhäuser, Rutishauser, \& Koch, 2008), is grounded in anatomical evidence about the primary locus of parkinsonian pathology (Agid et al., 1993; Kish, Shannak, \& Hornykiewicz, 1988; Farley, Price, \& Hornykiewicz, 1977), and parallels similar interpretations of identical performance patterns observed in monkeys with prefrontal neurochemical lesions (see below; Crofts et al., 2001).

The selective deficit in the low-salient condition is consistent with the robust finding that PD patients exhibit difficulty at the EDS stage of the original ID/ED set-shifting paradigm of the CANTAB battery, in which subjects have to shift from a highly salient shape dimension to a less salient line dimension (Cools, Barker, Sahakian, \& Robbins, 2001a; Owen et al., 1992, 1995; Downes et al., 1989). 
These prior studies had already revealed that a significant proportion of PD patients also failed to complete the early set formation (CD) stage of the task (e.g., Owen et al., 1992; Downes et al., 1989), already indicating that the deficit might reflect a failure of a more general (learning, selective attention, or control) mechanism than of set shifting specifically. Furthermore, it concurs with deficits on the Wisconsin Card Sorting Test (Grant \& Berg, 1948), a concept formation task, in which subjects are presented with three-dimensional stimuli. In this task, concept formation and shifting require the selection of one of two alternative, competing dimensions. The presence of a choice between dimensions might well reduce the salience (i.e., attention drawing capacity) of the newly relevant dimension. The data are also reminiscent of traditional accounts of set-shifting deficits, which attribute them to problems with "internal" attentional control, leading to excessive guidance of behavior by "external cues" (Van Spaendonck et al., 1995; Brown \& Marsden, 1988a; Cools et al., 1984; Barbeau, 1973). Specifically, several studies have indicated that PD patients exhibit greater difficulty with directing attention based on internal attentional cues than external attentional cues (Brown \& Marsden, 1990), not only in high-level cognitive tasks, as shown here, but also as measured with simple choice RT tasks and in the domain of movement (Jahanshahi et al., 1995). Here we provide direct evidence for the hypothesis that disproportionate control by bottom-up salience also accounts for the set-shifting deficit in PD; in fact, the deficit is observed only when control by bottom-up salience is detrimental for set shifting.

In the present study, the performance benefit for the high-salient over the low-salient dimension did not extend to performance at the preshift formation and/or maintenance stages (of the LI task). One might have predicted that disproportionate control by bottom-up attention would have also enhanced the impact of dimensional salience at the CD stage. In fact, although there was no significant Group $\times$ Condition interaction at the $\mathrm{CD}$ stage of the LI task, supplementary analyses revealed that a dimensional salience effect at the CD stage was significant only for PD patients, $F(1,50)=4.8, p=.03, \eta^{2}=0.09$, and not for controls, $F(1,21)=1.2, p=.3, \eta^{2}=0.05$. This post hoc analysis indicates that the predicted group effect at the CD stage may be present but simply not sufficiently large to reveal disproportionate control by bottom-up attention in patients relative to controls. This is perhaps not surprising, given that the preceding SD stages already forced selective attention to the relevant dimension, thus reducing the impact of differential salience of the irrelevant dimension. Indeed, prior data indicate that category learning in PD patients is impacted to a greater extent than controls when the number of randomly varying irrelevant dimensions increases (Filoteo, Maddox, Ing, Zizak, \& Song, 2005). Also consistent are findings that mild medicated PD patients are impaired in the identification of the dimensions of five-dimensional stimuli (Swainson et al.,
2006). Increases in the number of dimensions might well reduce the salience of each dimension.

The observation that responding was controlled by bottom-up dimensional salience to a greater extent in $\mathrm{PD}$, which is characterized by severe striatal dopamine depletion, apparently contrasts with the hypothesis that striatal dopamine promotes switching to salient, biologically relevant events (Redgrave, Prescott, \& Gurney, 1999). According to this hypothesis, PD should be characterized by reduced rather than enhanced ability to switch to salient information. One possibility is that our finding actually reflects enhanced striatal dopamine as a result of the dopaminergic medication taken by the majority of patients. This hypothesis would concur with current models of striatal dopamine function (Gruber, Dayan, Gutkin, \& Solla, 2006; Frank, 2005) and should be tested in a future controlled medication withdrawal study. However, attentional set shifting has previously been shown to be insensitive to dopaminergic medication in PD (Slabosz et al., 2006; Lewis et al., 2005; Cools et al., 2001a; Owen et al., 1993), which acts primarily by restoring striatal dopamine levels (Maruyama, Naoi, \& Narabayashi, 1996; Hornykiewicz, 1974). Therefore, we argue that it is more likely that the disproportionate control by bottom-up attention reflects an abnormality that does not involve striatal dopamine. Indeed there is evidence that different forms of switching implicate distinct cortical and subcortical mechanisms. Specifically, we have previously shown that the striatum is active and required only for switching between concrete stimulus exemplars but not for switching between abstract rules that have no direct instantiation in the motor or sensory domain (such as match or nonmatch rules) (Cools, Ivry, \& D'Esposito, 2006; Cools, Clark, \& Robbins, 2004). By contrast, the control of abstract (match and nonmatch) rules has been associated with prefrontal cortical mechanisms (Wallis, Anderson, \& Miller, 2001). Accordingly, the presently observed deficit in shifting between abstract dimensions may reflect an abnormality in nondopaminergic cortical mechanisms. Consistent with this hypothesis are recent data showing that mild PD patients are not impaired on a task-switching paradigm that requires the switching of both stimulus and response sets due to abstract rule changes (i.e., from judging letters as vowels or consonants to judging numbers as higher or lower than 5) (Kehagia, Cools, Barker, \& Robbins, 2009). This finding contrasted with results from our other task-switching studies (Cools, Barker, Sahakian, \& Robbins, 2003; Cools et al., 2001a, 2001b), which revealed consistent deficits in mild PD patients, when task-switching required the application of the same rule (i.e., naming) to different stimuli (i.e., letters or numbers), so that only the stimulus sets changed. Critically, dopaminergic medication withdrawal impaired the latter lower order stimulus set switching (Cools et al., 2001a, 2003) but did not affect the former higher order task-switching, in which both stimulus and response sets changed (Kehagia et al., 2009). Thus, not all forms of switching rely on corticostriatal dopamine 
transmission. Switching between abstract rules in these prior task-switching studies might be similar to extradimensional shifting in the context of the ID/ED paradigm. Indeed, findings from work with experimental animals have shown that EDS is sensitive to excitotoxic and neurochemical lesions in the pFC (McGaughy, Ross, \& Eichenbaum, 2008; Tait et al., 2007; Crofts et al., 2001; Birrell \& Brown, 2000; Roberts et al., 1994) and in the parietal cortex (Fox, Barense, \& Baxter, 2003) but not in the striatum (Crofts et al., 2001; Collins, Wilkinson, Everitt, Robbins, \& Roberts, 2000; for a review, see Robbins, 2007). In fact, the present performance pattern strikingly resembles that seen after catecholamine lesions in the pFC of nonhuman primates (Crofts et al., 2001). In marmosets, 6-hydroxydopamine lesions of PFC, which reduce both dopamine and noradrenaline levels there, induced abnormal responding that appeared disproportionately driven by bottom-up rather than top-down attention. Specifically, these lesions facilitated the ability to shift to a high-salient attentional set (shape) but not to a low-salient attentional set (line). Conversely, 6-hydroxydopamine lesions of the striatum left EDS in marmosets unaffected. Accordingly, the present parkinsonian failure of top-down control over attentional sets might be mediated by fronto-parietal cortical abnormalities in catecholamine (e.g., noradrenaline) neurotransmitter systems (Williams-Gray, Hampshire, Barker, \& Owen, 2008; Middleton, Sharma, Agouzoul, Sahakian, \& Robbins, 1999; Rogers et al., 1999) rather than striatal dopamine dysfunction.

These data provide a striking reinterpretation of previously observed parkinsonian set-shifting deficits, which were interpreted to reflect differential processing of previously present dimensions, that is, LI (Slabosz et al., 2006; Owen et al., 1993; but see Gauntlett-Gilbert et al., 1999; Van Spaendonck et al., 1995). We found that a group effect was present only in the PE task. In this task, it differed as a function of the salience of a novel, newly relevant dimension. Accordingly, the effect does not reflect disproportionate PE to a familiar, previously relevant dimension. In addition, there was no group effect in the LI task, indicating that it also does not reflect disproportionate LI of a previously irrelevant, now relevant dimension. Thus, the abnormality does not reflect a problem with shifting per se, but rather it reflects disproportionate control by bottom-up attention, which becomes evident most readily when tasks require shifting to a low-salient dimension. Indeed, the abnormality was expressed as an enhanced tendency to shift when the postshift dimension was high salient.

The lack of enhanced LI in the present study is apparently inconsistent with the previous observation that PD patients exhibited impairment on LI tasks (Slabosz et al., 2006; Owen et al., 1993). However, the hypothesis that $\mathrm{PD}$ is characterized by enhanced LI has long been controversial. Various other studies have obtained data inconsistent with this conclusion (Myers et al., 2003; Gauntlett-Gilbert et al., 1999; Van Spaendonck et al., 1995). In fact, Myers et al. (2003) have observed that PD patients (but not patients with medial-temporal lobe lesions) exhibit better rather than poorer shifting to a previously exposed (and irrelevant) dimension relative to a dimension to which subjects had not been exposed. Our data suggest that the previously observed effects of PD on LI tasks reflect effects of dimensional salience. Learned irrelevance usually reduces the salience of the previously irrelevant, now relevant dimension (Slabosz et al., 2006). It is possible that this effect did not surface in the present study (and other prior studies; e.g., Myers et al., 2003) because the preshift irrelevant dimension (and thus the postshift relevant dimension) was always "color"; this dimension was quite salient as shown by the sorting task data. In this case, prior exposure to a dimension, albeit irrelevant, might actually enhance rather than reduce its attention-drawing capacity (Myers et al., 2003). The fact that the newly relevant dimension in our LI tasks was always number, which was probably low salient, might have further attenuated a deficit in our LI tasks.

In conclusion, we replicated attentional set-shifting deficits, observed previously in PD. Unlike previous studies, the present study manipulated not only the need for overcoming LI but also dimensional salience and thus the need for top-down control. Results indicated that apparent setshifting deficits in PD do not reflect LI but rather impairments in response to low-salient information. These data should replicate in future studies, in which dimensional salience is manipulated using different dimensions and may reconcile apparently paradoxical findings that PD patients exhibit not only impaired attentional set shifting but also enhanced switching to and control by salient distracting cues in selective attention paradigms (Filoteo \& Maddox, 1999; Filoteo et al., 1994, 1997; Maddox, Filoteo, Delis, \& Salmon, 1996; Henik, Singh, Beckley, \& Rafal, 1993; Sharpe, 1990; Wright, Burns, Geffen, \& Geffen, 1990; Flowers \& Robertson, 1985). Intriguingly, the pattern of performance resembled that seen after catecholamine lesions in the $\mathrm{pFC}$ and provides the first direct evidence for exaggerated bottom-up and/or attenuated top-down attentional control during set shifting in PD.

\section{Acknowledgments}

This work was conducted within the Behavioural and Clinical Neuroscience Institute at the University of Cambridge, cofunded by the Medical Research Council and the Wellcome Trust. R. C. was supported by a Royal Society University Research fellowship. Thanks to Kirsty Beaton for assistance with data collection.

Reprint requests should be sent to Roshan Cools, Donders Institute for Brain, Cognition and Behaviour, Radboud University Nijmegen Medical Centre, The Netherlands, or via e-mail: roshan. cools@donders.ru.nl.

\section{REFERENCES}

Agid, Y., Ruberg, M., Javoy-Agid, F., Hirsch, E., Raisman-Vozari, R., Vyas, S., et al. (1993). Are dopaminergic neurons 
selectively vulnerable to Parkinson's disease? Advances in Neurology, 60, 148-164.

Barbeau, A. (1973). Biology of the striatum. In G. Gaull (Ed.), Biology of brain dysfunction (pp. 333-350). New York: Plenum Press.

Beck, A. T., Ward, C. H., Mendelson, M., Mock, J., \& Erbaugh, J. (1961). An inventory for measuring depression. Archives of General Psychiatry, 11, 561-571.

Birrell, J. M., \& Brown, V. J. (2000). Medial frontal cortex mediates perceptual attentional set shifting in the rat. Journal of Neuroscience, 20, 4320-4324.

Bowen, F. P., Kamienny, R. S., Burns, M. M., \& Yahr, M. D. (1975). Parkinsonism: Effects of levodopa treatment on concept formation. Neurology, 25, 701-704.

Brown, R., \& Marsden, C. (1990). Cognitive function in Parkinson's disease: From description to theory. Trends in Neurosciences, 13, 21-29.

Brown, R. G., \& Marsden, C. D. (1988a). Internal versus external cues and the control of attention in Parkinson's disease. Brain, 111, 323-345.

Brown, R. G., \& Marsden, C. (1988b). "Subcortical dementia": The neuropsychological evidence. Neuroscience, 25, 363-387.

Collins, P., Wilkinson, L. S., Everitt, B. J., Robbins, T. W., \& Roberts, A. C. (2000). The effect of dopamine depletion from the caudate nucleus of the common marmoset (Callithrix jacchus) on tests of prefrontal cognitive function. Behavioral Neuroscience, 114, 3-17.

Cools, A. R., Van Den Bercken, J. H. L., Horstink, M. W. I., Van Spaendonck, K. P. M., \& Berger, H. J. C. (1984). Cognitive and motor shifting aptitude disorder in Parkinson's disease. Journal of Neurology, Neurosurgery and Psychiatry, 47, 443-453.

Cools, R. (2006). Dopaminergic modulation of cognitive function-implications for L-DOPA treatment in Parkinson's disease. Neuroscience and Biobehavioral Reviews, 30, 1-23.

Cools, R., Barker, R. A., Sahakian, B. J., \& Robbins, T. W. (2001a). Enhanced or impaired cognitive function in Parkinson's disease as a function of dopaminergic medication and task demands. Cerebral Cortex, 11, 1136-1143.

Cools, R., Barker, R. A., Sahakian, B. J., \& Robbins, T. W. (2001b). Mechanisms of cognitive set flexibility in Parkinson's disease. Brain, 124, 2503-2512.

Cools, R., Barker, R. A., Sahakian, B. J., \& Robbins, T. W. (2003). L-Dopa medication remediates cognitive inflexibility, but increases impulsivity in patients with Parkinson's disease. Neuropsychologia, 41, 1431-1441.

Cools, R., Clark, L., \& Robbins, T. W. (2004). Differential responses in human striatum and prefrontal cortex to changes in object and rule relevance. Journal of Neuroscience, 24, 1129-1135.

Cools, R., Ivry, R., \& D'Esposito, M. (2006). The human striatum is necessary for responding to changes in stimulus relevance. Journal of Cognitive Neuroscience, 18, 1959-1972.

Crofts, H. S., Dalley, J. W., Van Denderen, J. C. M., Everitt, B. J., Robbins, T. W., \& Roberts, A. C. (2001). Differential effects of 6-OHDA lesions of the frontal cortex and caudate nucleus on the ability to acquire an attentional set. Cerebral Cortex, 11, 1015-1026.

Dias, R., Robbins, T. W., \& Roberts, A. C. (1996). Dissociation in prefrontal cortex of affective and attentional shifts. Nature, 380, 69-72.

Downes, J. J., Roberts, A. C., Sahakian, B. J., Evenden, J. L., Morris, R. G., \& Robbins, T. W. (1989). Impaired extra-dimensional shift performance in medicated and unmedicated Parkinson's disease: Evidence for a specific attentional dysfunction. Neuropsychologia, 27, 1329-1343

Dubois, B., Pillon, B., Malapani, C., Deweer, B., Verin, M., Partiaud, A., et al. (1995). Subcortical dementia and Parkinson's disease: What are the cognitive functions of the basal ganglia? In F. Boller \& J. Grafman (Eds.), Handbook of neuropsychology (pp. 195-240). New York: Elsevier.

Einhäuser, W., Rutishauser, U., \& Koch, C. (2008). Task-demands can immediately reverse the effects of sensory-driven saliency in complex visual stimuli. Journal of Vision, 8, 2.1-2.19.

Farley, I., Price, K., \& Hornykiewicz, O. (1977). Dopamine in the limbic regions of the human brain: Normal and abnormal. Advances in Biochemical Psychopharmacology, $16,57-64$.

Filoteo, J., Maddox, W., Ing, A., Zizak, V., \& Song, D. (2005). The impact of irrelevant dimensional variation on rule-based category learning in patients with Parkinson's disease. Journal of the International Neuropsychological Society, $11,503-513$.

Filoteo, J. V., Delis, D. C., Demadura, T. L., Salmon, D. P., Roman, M. J., \& Shults, C. W. (1994). Abnormally rapid disengagement of covert attention to global and local stimulus levels may underlie the visuoperceptual impairment in Parkinson's patients. Neuropsychology, 8, 218-226.

Filoteo, J. V., Delis, D. C., Salmon, D. P., Demadura, T., Roman, M. J., \& Shults, C. W. (1997). An examination of the nature of attentional deficits in patients with Parkinson's disease: Evidence from a spatial orienting task. Journal of the International Neuropsychological Society, 3, 337-347.

Filoteo, J. V., \& Maddox, W. T. (1999). Quantitative modeling of visual attention processes in patients with Parkinson's disease: Effects of stimulus integrality on selective attention and dimensional integration. Neuropsychology, 13, 106-222.

Flowers, K. A., \& Robertson, C. (1985). The effect of Parkinson's disease on the ability to maintain a mental set. Journal of Neurology, Neurosurgery and Psychiatry, 48, 517-529.

Folstein, M. F., Folstein, S. E., \& McHugh, P. R. (1975). "Mini Mental State": A practical method for grading the cognitive state of patients for the clinician. Journal of Psychiatric Research, 12, 189-198.

Fox, M. T., Barense, M. D., \& Baxter, M. G. (2003). Perceptual attentional set-shifting is impaired in rats with neurotoxic lesions of posterior parietal cortex. Journal of Neuroscience, 23, 676-681.

Frank, M. J. (2005). Dynamic dopamine modulation in the basal ganglia: A neurocomputational account of cognitive deficits in medicated and nonmedicated Parkinsonism. Journal of Cognitive Neuroscience, 17, 51-72.

Garner, W., \& Felfoldy, G. (1970). Integrality of stimulus dimensions in various types of information processing Cognitive Psychology, 1, 225-241.

Gauntlett-Gilbert, J., Roberts, R. C., \& Brown, V. J. (1999). Mechanisms underlying attentional set-shifting in Parkinson's disease. Neuropsychologia, 37, 605-616.

Gazzaley, A., \& D'Esposito, M. (2007). Top-down modulation and normal aging. Annals of the New York Academy of Sciences, 1097, 67-83.

Gottwald, R., \& Garner, W. (1975). Filtering and condensation tasks with integral and separable dimensions. Perception Psychophysics, 18, 26-28. 
Grant, D. A., \& Berg, E. A. (1948). A behavioural analysis of degree of reinforcement and ease of shifting to new responses in a Weigl-type card sorting problem. Journal of Experimental Psychology, 38, 404-411.

Gruber, A. J., Dayan, P., Gutkin, B. S., \& Solla, S. A. (2006). Dopamine modulation in the basal ganglia locks the gate to working memory. Journal of Computational Neuroscience, 20, 153-166.

Henik, A., Singh, J., Beckley, D. J., \& Rafal, R. D. (1993). Disinhibition of automatic word reading in Parkinson's disease. Neurology, 17, 424-427.

Hornykiewicz, O. (1974). The mechanisms of action of L-DOPA in Parkinson's disease. Life Sciences, 15, 1249-1259.

Howell, D. C. (1997). Statistical methods for psychology. Belmont: Wadsworth Publishing Company.

Jahanshahi, M., Jenkins, I. H., Brown, R. G., Marsden, C. D., Passingham, R. E., \& Brooks, D. J. (1995). Self-initiated versus externally triggered movements. An investigation using measurement of regional cerebral blood flow with PET and movement-related potentials in normal and Parkinson's disease subjects. Brain, 118, 913-933.

Kehagia, A., Cools, R., Barker, R. A., \& Robbins, T. (2009). Switching between abstract rules reflects disease severity but not dopaminergic status in Parkinson's disease. Neuropsychology, 47, 1117-1127.

Kish, S. J., Shannak, K., \& Hornykiewicz, O. (1988). Uneven patterns of dopamine loss in the striatum of patients with idiopathic Parkinson's disease. New England Journal of Medicine, 318, 876-880.

Lees, A. J., \& Smith, E. (1983). Cognitive deficits in the early stages of Parkinson's disease. Brain, 106, 257-270.

Lewis, S., Slabosz, A., Robbins, T., Barker, R., \& Owen, A. (2005). Dopaminergic basis for deficits in working memory but not attentional set-shifting in Parkinson's disease. Neuropsychologia, 43, 823-832.

Mackintosh, N. (1975). A theory of attention: Variations in the associability of stimulus with reinforcement. Psychological Review, 82, 276-298.

Maddox, W. T., Filoteo, J. V., Delis, D. C., \& Salmon, D. P (1996). Visual selective attention deficits in patients with Parkinson's disease: A quantitative model-based approach. Neuropsychology, 10, 197-218.

Maruyama, W., Naoi, M., \& Narabayashi, H. (1996).

The metabolism of L-DOPA and L-threo-3,4dihydroxyphenylserine and their effects on monoamines in the human brain: Analysis of the intraventricular fluid from parkinsonian patients. Journal of the Neurological Sciences, 139, 141-148.

McGaughy, J., Ross, R. S., \& Eichenbaum, H. (2008). Noradrenergic, but not cholinergic, deafferentation of prefrontal cortex impairs attentional set-shifting. Neuroscience, 53, 63-71.

Middleton, H. C., Sharma, A., Agouzoul, D., Sahakian, B. J., \& Robbins, T. W. (1999). Idoxan potentiates rather than antagonizes some of the cognitive effects of clonidine. Psychopharmacology, 145, 401-411.

Miller, B. T., \& D'Esposito, M. (2005). Searching for "the top" in top-down control. Neuron, 48, 535-538.

Miller, E., \& Cohen, J. (2001). An integrative theory of prefrontal cortex function. Annual Review of Neuroscience, 24, 167-202.

Milner, B. (1963). Effects of different brain lesions on card sorting. Archives of Neurology, 9, 100-110.

Milner, B. (1964). Some effects of frontal lobectomy in man. In J. M. Warren and K. Akert (Eds.), The frontal granular cortex and behaviour (pp. 410-444). New York: McGraw-Hill.

Myers, C., Shohamy, D., Gluck, M., Grossman, S., Kluger, A., Ferris, S., et al. (2003). Dissociating hippocampal versus basal ganglia contributions to learning and transfer. Journal of Cognitive Neuroscience, 15, 185-193.

Nelson, H. E. (1982). National Adult Reading Test (NART) Test Manual. Windsor, UK: NFER-Nelson.

Owen, A. M., James, M., Leigh, J. M., Summers, B. A., Marsden, C. D., Quinn, N. P., et al. (1992). Fronto-striatal cognitive deficits at different stages of Parkinson's disease. Brain, 115, 1727-1751.

Owen, A. M., Roberts, A. C., Hodges, J. R., Summers, B. A., Polkey, C. E., \& Robbins, T. W. (1993). Contrasting mechanisms of impaired attentional set-shifting in patients with frontal lobe damage or Parkinson's disease. Brain, 116, 1159-1179.

Owen, A. M., Roberts, A. C., Polkey, C. E., Sahakian, B. J., \& Robbins, T. W. (1991). Extra-dimensional versus intra-dimensional set-shifting performance following frontal lobe excisions, temporal lobe excisions or amygdalo-hippocampectomy in man. Neuropsychologia, 29, 993-1006.

Owen, A. M., Sahakian, B. J., Hodges, J. R., Summers, B. A., Polkey, C. E., \& Robbins, T. W. (1995). Dopamine-dependent frontostriatal planning deficits in early Parkinson's disease. Neuropsychology, 9, 126-140.

Redgrave, P., Prescott, T. J., \& Gurney, K. (1999). The basal ganglia: A vertebrate solution to the selection problem? Neuroscience, 89, 1009-1023.

Robbins, T. (2007). Shifting and stopping: Fronto-striatal substrates, neurochemical modulation and clinical implications. Philosophical Transactions of the Royal Society of London, Series B, Biological Sciences, 362, 917-932.

Roberts, A. C., De Salvia, M. A., Wilkinson, L. S., Collins, P., Muir, J. L., Everitt, B. J., et al. (1994). 6-Hydroxydopamine lesions of the prefrontal cortex in monkeys enhance performance on an analog of the Wisconsin Card Sort Test: Possible interactions with subcortical dopamine. Journal of Neuroscience, 14, 2531-2544.

Rogers, R. D., Blackshaw, A. J., Middleton, H. C., Matthews, K., Hawtin, K., Crowley, C., et al. (1999). Tryptophan depletion impairs stimulus-reward learning while methylphenidate disrupts attentional control in healthy young adults: Implications for the monoaminergic basis of impulsive behaviour. Psychopharmacology, 146, 482-491.

Sahakian, B. J., Morris, R. G., Evenden, J. L., Heald, A., Levy, R., Philpot, M., et al. (1988). A comparative study of visuospatial memory and learning in Alzheimer-type dementia and Parkinson's disease. Brain, 111, 695-718.

Sharpe, H. S. (1990). Distractibility in early Parkinson's disease. Cortex, 26, 239-246.

Slabosz, A., Lewis, S. J., Smigasiewicz, K., Szymura, B., Barker, R. A., \& Owen, A. M. (2006). The role of learned irrelevance in attentional set-shifting impairments in Parkinson's disease. Neuropsychology, 20, 578-588.

Swainson, R., SenGupta, D., Shetty, T., Watkins, L. H., Summers, B. A., Sahakian, B. J., et al. (2006). Impaired dimensional selection but intact use of reward feedback during visual discrimination learning in Parkinson's disease. Neuropsychologia, 44, 1290-1304.

Tait, D. S., Brown, V. J., Farovik, A., Theobald, D. E., Dalley, J. W., \& Robbins, T. W. (2007). Lesions of the dorsal noradrenergic bundle impair attentional set-shifting in 
the rat. European Journal of Neuroscience, 25, 3719-3724.

Taylor, A. E., Saint-Cyr, J. A., \& Lang, A. E. (1986). Frontal lobe dysfunction in Parkinson's disease. Brain, 109, 845-883.

Van Spaendonck, K. P. M., Berger, H. J. C., Horstink, M. W. I., Borm, G. F., \& Cools, A. R. (1995). Card sorting performance in Parkinson's disease: A comparison between acquisition and shifting performance. Journal of Clinical and Experimental Neuropsychology, 17, 918-925.
Wallis, J., Anderson, K., \& Miller, E. (2001). Single neurons in prefrontal cortex encode abstract rules. Nature, 411, 953-956.

Williams-Gray, C. H., Hampshire, A., Barker, R. A., \& Owen, A. M. (2008). Attentional control in Parkinson's disease is dependent on COMT val 158 met genotype. Brain, 131, 397-408.

Wright, M. J., Burns, R. J., Geffen, G. M., \& Geffen, L. B. (1990). Covert orientation of visual attention in Parkinson's disease: An impairment in the maintenance of attention. Neuropsychologia, 28, 151-159. 